\title{
SF6 High Voltage Circuit Breaker Contact Systems Under Lightning Impulse and Very Fast Transient Voltage Stress
}

\author{
Journal Article \\ Author(s): \\ Simka, Philipp; Straumann, Ulrich; Franck, Christian (D) \\ Publication date: \\ 2012-06 \\ Permanent link: \\ https://doi.org/10.3929/ethz-b-000051187
}

Rights / license:

In Copyright - Non-Commercial Use Permitted

Originally published in:

IEEE Transactions on Dielectrics and Electrical Insulation 19(3), https://doi.org/10.1109/TDEl.2012.6215088 


\section{2011 IEEE.}

"Personal use of this material is permitted. Permission from IEEE must be obtained for all other uses, in any current or future media, including reprinting/republishing this material for advertising or promotional purposes, creating new collective works, for resale or redistribution to servers or lists, or reuse of any copyrighted component of this work in other works."

Digital Object Identifier: http://dx.doi.org/10.1109/TDEl.2012.6215088 


\title{
$\mathrm{SF}_{6}$ High Voltage Circuit Breaker Contact Systems Under Lightning Impulse and Very Fast Transient Voltage Stress
}

\author{
P. Simka, U. Straumann and C. M. Franck \\ Power Systems and High Voltage Laboratories, ETH Zurich, 8092 Zurich, Switzerland
}

\begin{abstract}
In the past few years gas insulated high voltage circuit breakers have improved considerably, in particular with respect to required drive energy for operation, compactness and reliability. A further reduction in size implies increasing operational field stress inside the breaker and, thus, a reduction in built-in safety margins. If the design comes closer to its physical limits, in particular the dielectric coordination of the contact systems becomes more and more challenging. The aim of the present work is to identify factors that may influence the dielectric coordination of contact systems with reduced coordination margin. The breakdown probability distribution of $\mathrm{SF}_{6}$ insulated multi-contact systems was investigated with the focus on two aspects. First of all, the breakdown probabilities under lightning impulse (LI) stress were measured. These indicated that it is not feasible to extrapolate the breakdown probability curves of single contacts to a combined full contact system. Secondly, the findings on the same contact system stressed with very fast transient voltages (VFT) showed a considerable reduction in breakdown voltage and suggested a significant increase in the main contact breakdown probability, compared to the LI tests. The results and interpretation within this work indentified the polarity and voltage shape dependent breakdown initiation of different contacts as causes for circuit breaker contact system failure.
\end{abstract}

Index Terms $-\mathbf{S F}_{6}$ high voltage gas circuit breakers, breaker contacts, electric fields, dielectric breakdown, breakdown probability, switching transients, very fast transients.

\section{INTRODUCTION}

HIGH voltage gas circuit breakers are the key safety devices in electric power systems and have to separate faulty parts of the grid quickly and reliably. The interruption capability of high voltage circuit breakers have been increased over the last decades and today, $\mathrm{SF}_{6}$ insulated circuit breakers are capable of switching up to 20 GVA per interrupting unit [1].

Today's circuit breakers have two different contact systems: the main (nominal) and the arcing contact system. The outer main contact system is optimized for minimum contact resistance in order to carry currents with low losses in the closed position, whereas the inner arcing contact system is designed to carry switching arcs. The breaker itself and its nozzle system are designed to quench and interrupt an arc burning between the arcing contacts. It must be ensured that any breakdown of the contact gap and all switching arcs only occur on the arcing contacts. The associated design procedure is known as dielectric coordination. Typically, this is achieved by exposing the arcing contacts in axial direction with respect to the main contacts. When the circuit breaker opens, the main contacts separate first and the current is commutated from the main to the arcing contacts. Later, when the arcing contacts separate, the switching arc is formed and burns between them. In a closing operation, the two contact systems approach each other and the gas gap between them breaks down, also called pre-strike. The axial exposure of the arcing contacts with respect to the main contacts leads to an increased electric field stress and ensures breakdown (and subsequent arc initiation) between the arcing contacts. Arcs on the main contact during breaker opening must be avoided under any circumstances as they cannot be extinguished and could lead to a complete breaker failure. An arc initiated by a breakdown between the main contacts during breaker closing would extinguish when the contacts touch, but could still lead to damage of the insulator and must also be avoided.

Dielectric coordination is usually a compromise between strong electric field enhancement on the arcing contacts to promote the breakdown initiation between them, minimum field enhancement to increase the voltage withstand of the open breaker, and a minimum breaker size. To enable further circuit breaker design optimization, the following questions are investigation here: 
- Which phenomena beyond the electrostatic field distribution are important for the dielectric coordination of circuit breaker contact systems?

- Is it possible to calculate breakdown voltages reliably?

- Is it possible to extrapolate (lightning impulse) breakdown probability distribution functions from single contact measurements to full contact systems?

- Are dielectric coordination failures more frequent if very fast transients (VFT) exist compared to LI voltages, only?

These questions are investigated on a specific simplified circuit breaker model with typical contact configurations and common coordination margins. Even though this does not permit to draw universally valid conclusions, insights into the questions mentioned above can be given.

This paper is an extension of the data presented in [3] and it is structured as follows: In Section 2, the theoretical basis for breakdown in low inhomogeneous, $\mathrm{SF}_{6}$ insulated electrode arrangements is reviewed and the most important mathematical tools are introduced. Section 3 describes the experimental method; that is the setup, diagnostics, and the evaluation procedure. Section 4 presents the results of both, the LI and VFT experiments as well as the results of the breakdown voltage calculations, which are discussed in Section 5. Section 6 concludes this paper with a summary of the key findings.

\section{BREAKDOWN IN SF 6}

A breakdown in the gas gap between two contacts with a homogeneous (or slightly inhomogeneous) electric field distribution can occur (necessary condition) if the critical electric field strength $(E / p)_{\text {crit }}$ is exceeded in some places and if the streamer criterion is fulfilled [4]. For typical filling pressures of gas insulated switchgear $\left(p_{\text {fill }}=0.4-0.6 \mathrm{MPa}(4-6\right.$ bar $)$ ), the streamer criterion is fulfilled on very short lengths of a few $100 \mu \mathrm{m}$ or lower in the frequent case, where electrode roughness determines the breakdown voltage $[4,5]$. Therefore, a simpler and more practical criterion is often used for $\mathrm{SF}_{6}$ gas circuit breaker design: the maximal macroscopic field strength $E_{\text {max }}$ on an electrode surface.

The streamer criterion is sufficient with slowly rising voltages (ac and dc) in homogeneous or technically relevant (weakly inhomogeneous) configurations, but it is observed that the breakdown voltages are typically higher with impulse voltage stress. In addition, a first electron must be available in the critical region to start the avalanche. For impulse voltage stresses of short duration this may not apply and the statistical time-lag of the first electron creation becomes relevant $[6,7]$.

If the polarity of the electrode with higher electric field stress is negative, such first electrons are likely to be produced by field emission [8]. A microscopic electric field of $1 \mathrm{MV} / \mathrm{cm}$ [4] up to $10 \mathrm{MV} / \mathrm{cm}$ [9] has to be present in order to enable this process. Such field stress can be reached at the electrode surface even in low inhomogeneous field configurations due to small surface defects or electrode roughness. The rate of electrons leaving the electrode due to field emission can be calculated by the FowlerNordheim equation as in [10].

At positive polarity, the dominant mechanism to generate first electrons is collisional electron detachment from $\mathrm{SF}_{6}^{-}$ ions [11]. To judge the breakdown probability $P$ of gas gaps with low inhomogeneous electric field distribution and different sizes, the volume-time criterion can be applied [7, 12]. It is calculated using the electron production rate $\frac{\mathrm{d} n_{\mathrm{e}}}{\mathrm{dt}}$ and the ionization $\alpha$ and recombination coefficients $\eta$, with

$$
P(t)=1-\exp \left[-\int_{0}^{t} \iiint \frac{\mathrm{d} n_{\mathrm{e}}}{\mathrm{dt}}\left(1-\frac{\eta}{\alpha}\right) \mathrm{dV}_{\mathrm{cr}} \mathrm{dt}\right] .
$$

The volume-time criterion accounts for the fact that different statistical time lags occur for different electric field stresses in volumes of different sizes. Generally speaking, the statistical time lag decreases with increasing volume exceeding the critical field strength $\left(\mathrm{V}_{\mathrm{cr}}\right)$.

Consequently, the impulse breakdown voltages differ significantly for negative and positive voltage polarity in such situations. Besides longer statistical time lags in the case of positive polarity, the time to arc formation is also influenced by the formative time-lag [10]. The time scales of this formative time-lag are larger for negative polarity [13].

It was observed that the breakdown voltages are smaller if the insulating gas gap is stressed with VFT voltages compared to LI $[14,15]$. The transition of a streamer discharge to a conductive leader channel can be supported by high frequency voltages $U(t)$ of sufficient amplitude. The streamer volume is heated by the high frequency displacement currents $i(t)[15]$ :

$$
i(t)=C(t) \frac{\mathrm{d} U(t)}{\mathrm{dt}}+U(t) \frac{\mathrm{d} C(t)}{\mathrm{dt}} .
$$

In summary, VFT voltages may have three different effects relevant to the present investigation: Firstly, an increase of voltage stress through constructive reflection of travelling waves within the gas insulated system. Secondly, the potential field distribution may differ from the electrostatic one and there could be a voltage difference between the main and arcing contacts. Thirdly, the breakdown voltage may be reduced by the highfrequency mechanism described above.

\section{EXPERIMENTAL APPROACH}

In order to investigate a possible failure of the dielectric coordination, a contact system similar to that of a circuit breaker was designed and constructed. In the following this specimen is referred to simply as the 'contact system'. The macroscopic electrostatic field distributions were calculated and the breakdown probability of single contacts and the complete contact system was measured using lightning impulse voltages [16] and very fast transient voltages superimposed on lightning impulse.

\subsection{GEOMETRY OF CONTACT SYSTEM}

Five different electrodes were used to investigate the breakdown behavior as depicted in Figure 1. A plane electrode and four contacts whose geometry is similar in size and curvature to those used in high voltage circuit breakers were used for all experiments. The electrodes are made from stainless steel $(\mathrm{NiCr})$ and the tips are spherically rounded. The contacts are only used to study the breakdown behavior, i.e. they are not designed to carry current or withstand arcing. The tulip contact, for example, is thus designed as a hollow cylinder with rounded front sides. The contacts do 
not support a nozzle as no arc quenching capability is needed. The contacts are fixed during the experiments but the axial position of each of the four contacts can be adjusted individually. By this, there is the flexibility to expose the contacts to different electric fields. Thus, the dielectric coordination in specific situations without the influence of the movement of the contacts can be investigated. The surfaces of the electrodes were sandblasted $(80 \mu \mathrm{m}$ grain size) in order to minimize the relative change in surface condition during the experiments. This treatment resulted in a mean surface roughness of $R_{a}=1.85 \mu \mathrm{m}$ and a maximum surface roughness of $R_{z}=15.22 \mu \mathrm{m}$ (measured according to ISO 4288:1996E).

In analogy to a real circuit breaker, the contact system with the plug is hereafter called fixed contact system and the one with the tulip moving contact system.
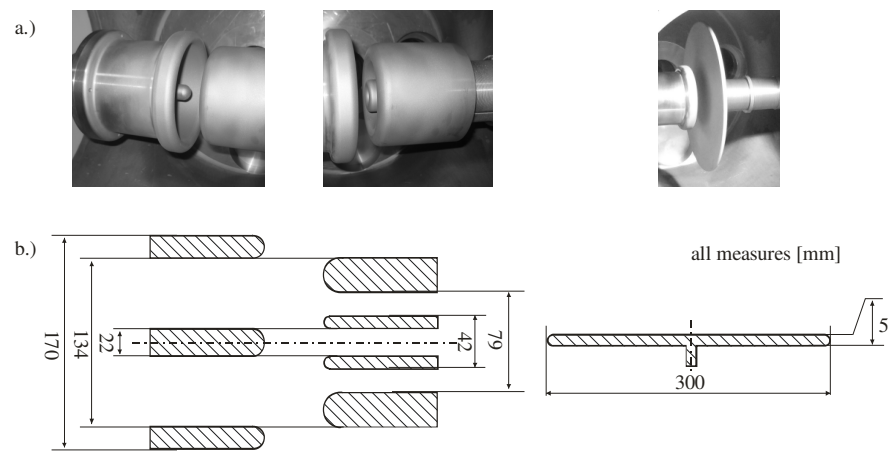

Figure 1. Photographs a.) and schematic drawing and dimensions b.) of the contacts used in the experiments. The sandblasted stainless steel electrodes represent a high voltage circuit breaker contact system. From left to right: Fixed contact system with main contact (ring), arcing contact (plug), moving contact system with main contact (ring) and arcing contact (tulip) and plane electrode.

Figure 2 shows the macroscopic electrostatic field distribution of the contact system, calculated with Comsol Multiphysics 3.4. The tips of the arcing and nominal contacts are aligned in axial direction. It can be seen that the electric field strength for this configuration is largest at the fixed arcing contact (plug); it is almost twice as high compared to the other electrodes, which is to assure arcing to happen on this contact.
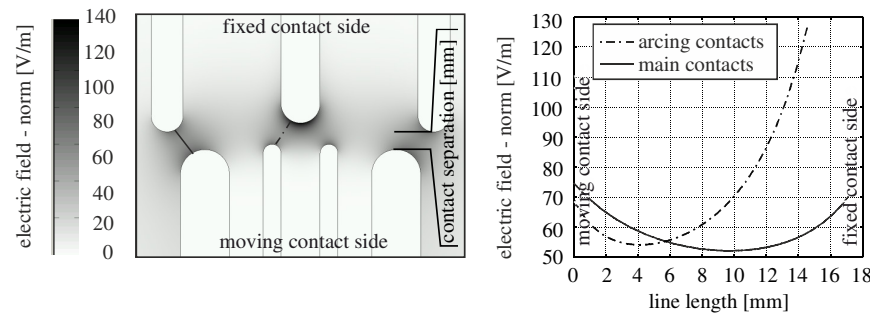

Figure 2. Electrostatic field of the contact system, calculated with $1 \mathrm{~V}$ potential difference and $10 \mathrm{~mm}$ contact separation between fixed and moving contact side. Electric field distribution (left) and electric field along the shortest distance (right).

\subsection{EXPERIMENTAL SETUP}

The experimental setup used to determine the breakdown probability of the specimen is shown in Figure 3. The voltage source was a Marx Generator with maximum charging voltage of $1 \mathrm{MV}$. The test vessel was a standard
$\mathrm{SF}_{6}$-filled GIS element attached via disconnector, earthing switch, and gas insulated bushing to the air insulated parts on the source side. The load side was grounded with a $2.2 \mathrm{M} \Omega$ resistor (and, in parallel, with the stray capacitance of the connecting GIS-circuit). The resistor is inserted in order to minimize the energy impact on the electrodes in the case of a breakdown.
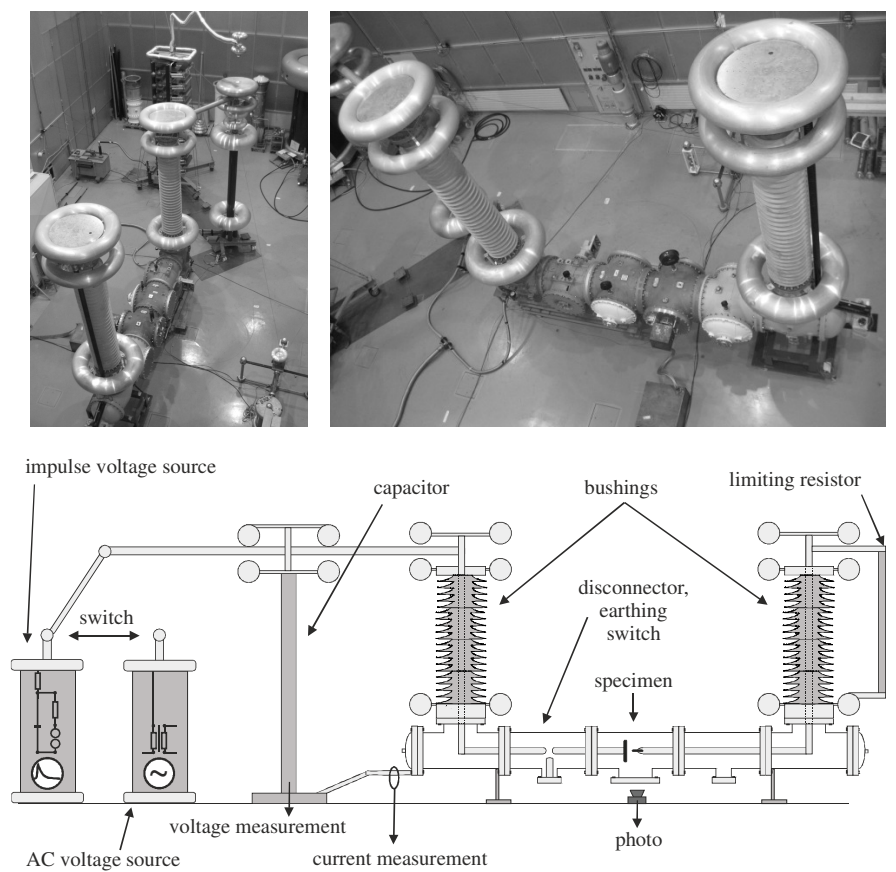

Figure 3. Picture and schematic drawing of the experimental setup used to evaluate breakdown probabilities of $\mathrm{SF}_{6}$ insulated high voltage circuit breaker contacts used in the investigations.

An ac voltage source was used to sweep excessive ions in between the individual breakdown experiments in order to reach a reproducible condition of the gas and to ensure statistical independence of the experimental series [17]. Consequently, no long waiting time in between the measurements was needed.

\subsection{DIAGNOSTICS}

The applied voltage was measured using a series damped capacitive voltage divider (cf. also Figure 3). The current across the electrodes was measured with a Pearson current monitor, attached in the ground return path of the GIS encapsulation. Both measurements were recorded with a digital storage oscilloscope (LeCroy, Wavepro7000, $1 \mathrm{GHz}$, $10 \mathrm{Gs} / \mathrm{s})$.

In order to determine whether a breakdown happened on the arcing or the main contact, each shot was photographed by a digital single-lens reflex camera (Nikon D50). In addition to the direct image, the camera also recorded the image from two mirrors installed inside the GIS. By means of triangulation methods the exact location of the arc root on the contacts was determined $[3,18]$.

\subsection{EXPERIMENTAL PROCEDURE}

During the assembly the specimen and the test vessel were kept free of dust, and contact with skin was omitted to prevent pollution of the electrodes such as deposition of 
grease in order to minimize relative change of surface condition during the experiments. The test vessel was then evacuated (70 $\mathrm{Pa}=0.7 \mathrm{mbar})$ and filled with $\mathrm{SF}_{6}$ at $0.6 \mathrm{MPa}$ (6 bar) absolute pressure.

After each application of a voltage impulse, irrespective of whether a breakdown had occurred or not, the ac voltage source was connected for $30 \mathrm{~s}$ with voltage amplitude of 5 $10 \%$ of the peak impulse voltage. By this, all ions between the contacts were removed. After this, it took $60 \mathrm{~s}$ to disconnect the ac source and to charge the impulse voltage generator. Applying this procedure in exactly the same way for each measurement ensures a reproducible ion distribution and thus also statistical independence of the experiment. Changing of the contacts due to arcing foot points could impair statistical independence as well. However, minimizing the impact of arcing with the resistor seemed sufficient, as mathematical tests revealed statistical independence of the experimental series. These tests consisted of the iteration-test and the test of comparison of breakdown probabilities [17].

The breakdown probability of the contacts was determined by using the constant voltage method according to [16].

\subsection{EVALUATION PROCEDURE}

A typical waveform of a breakdown measured under applied lightning impulse is depicted in Figure 4. A breakdown across the electrodes happened at approximately $1.3 \mu$ s. The time to breakdown was calculated between $10 \%$ of the prospective peak voltage amplitude and the actual breakdown. The peak voltage of the measured signal, the occurrence of and the time to breakdown was determined and stored for post processing. Noteworthy, the breakdown in Figure 4 did not lead to an instantaneous discharge of the Marx Generator, indicating that the resistor attached on the load side provided an effective current limitation. This extinguished the spark and the source capacitors of the impulse generators discharged through the source resistors. Hence, the energy input and thus the damage to the electrodes is effectively reduced. This supports the statistical independence with respect to the electrode surface of the measurement series.
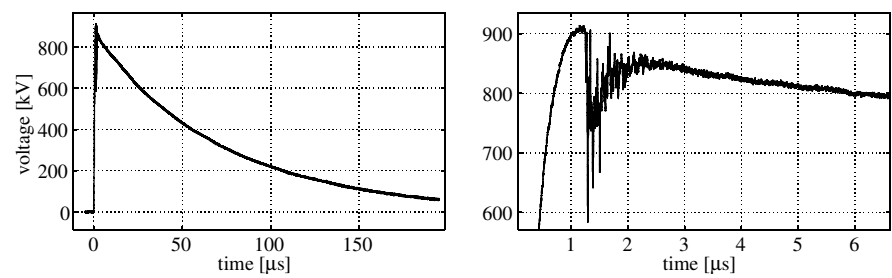

Figure 4. Voltage measurement of lightning impulse voltage application on the model contact system with an amplitude of approximately $900 \mathrm{kV}$ (left) and its detail in the range of breakdown (right).

The generation of VFT stressing the contact system was reached by opening the disconnector of the experimental setup (cf. Figure 3). The voltage application resulted in a preignition across the open disconnector which induced travelling waves in the compartment between disconnector and specimen. The corresponding measurement is shown in
Figure 5. Two subsequent oscillations were recorded. The first one, starting at $1.2 \mu \mathrm{s}$, represented the pre-ignition across the disconnector contacts, whereas the second oscillation at about $1.8 \mu \mathrm{s}$ was the breakdown across the circuit breaker contacts. Notably, the recorded peak amplitude in Figure 5 was $600 \mathrm{kV}$ and thus considerably higher than the prospective lightning impulse voltage of $520 \mathrm{kV}$. Furthermore, the measurement system was not optimized for measuring high frequency. Hence, the peak amplitude stressing the contact system is assumed to be higher than the measured one. However, some information on such peak amplitudes is given by calculation. According to the simulation guidelines presented in $[20,21]$ time resolved simulations of the experimental geometry were made in addition to the experiments [3]. The frequency content of the excited oscillation is similar to the one measured and shown in Figure 5. From the simulations it can be seen that the peak voltage stress at the contact system is $80 \%$ higher compared to the pure LI stress.

The disconnector was closed during the application of the ac voltage (cf. section 2.4) and reopened before impulse voltage application.
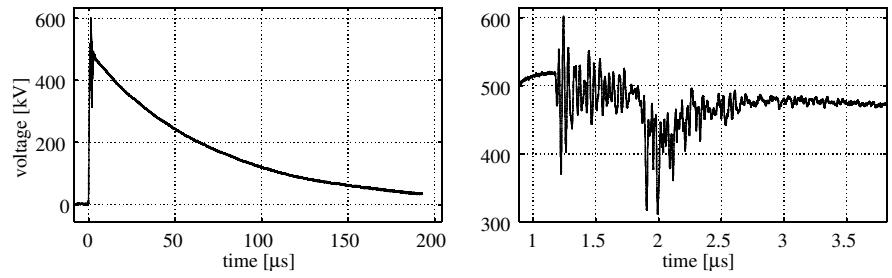

Figure 5. Voltage measurement of VFT voltage superimposed on lightning impulse voltage shape stressing the contact system (left) and its detail in the range of breakdown at $1.8 \mu$ s (right).

The empirical breakdown probability curve of the measured peak voltages was approximated by a normal distribution and the $95 \%$ confidence intervals were calculated as well as the standard deviation $\sigma$ and the mean value of the distribution $U_{50}$.

To compare these values with the theoretical breakdown voltage, the latter was calculated by using the ideal streamer criterion (2.1) as well as the volume-time criterion (2.2). Both were evaluated for different voltages, scaling the electric field values obtained from the FEM-calculations. Approximations for $\alpha$ and $\eta$ were taken from [5]. The streamer criterion (2.1) was evaluated for $\mathrm{k}_{\mathrm{st}}=18.5$ (according to [5]).

In order to calculate the volume-time criterion, the normalized measured voltage shapes from Figures 4 and 5 were used to scale the electric field values. The temporal integration boundaries were set between $\mathrm{t}=0$ and $300 \mu \mathrm{s}$, consequently integrating the whole voltage shape. The volume $V_{\text {cr }}$ for the space integration consisted of the region with $(E / p) \geq(E / p)_{\text {crit }}$ and $d \geq x_{\text {crit }}$ with $d$ being the minimum distance from the electrode surface. This calculation was repeated for the necessary voltage range in order to obtain the breakdown probability curve dependent on the voltage. The 50\%-value of this curve will be given from now on. The electron production rate $\frac{d n_{e}}{d t}$ was calculated using the 
electron detachment ratio from [19] and calculated ion density of $1905 \mathrm{IP} / \mathrm{cm}^{3}$ - corresponding to $60 \mathrm{~s}$ of ion pair (IP) generation by radiation at a rate of $32 \mathrm{IP} /\left(\mathrm{cm}^{3} \mathrm{~s}\right)$ at 0.6 $\mathrm{MPa}$ (6 bar). Latter has been measured in the laboratory, which is $10 \mathrm{~m}$ below ground level (compared to 26$55 \mathrm{IP} /\left(\mathrm{cm}^{3} \mathrm{~s}\right)$ at $0.4 \mathrm{MPa}$ (4 bar) in [7]).

To calculate the streamer inception triggered by field emission on negative polarity the analogue procedure was executed. The relevant changes are that in the volume-time criterion (2.2) the volume was replaced by the electrode surface and the electron production rate is given by field emission. For latter the formula from [5] has been used.

Even though it was measured, the surface roughness is not well defined and choosing protrusions to represent the roughness in the quantitative evaluation of the breakdown criteria certainly offers some variety. But, the measurement gives some limits for such protrusions. Further, not all experimental behavior can be reproduced by the appropriate matching choice of such protrusions; with the choice of the protrusions, the breakdown probability distribution of one situation can be reproduced, the consistence with the breakdown behavior of the other situation is then only given by the criteria. Therefore, such including of surface roughness, reveals, whether the breakdown criteria and the underlying physics is the appropriate and the decisive for the breakdown process. This is why the surface roughness is taken into account in the following by choosing some type of protrusions.

To account for the surface roughness the field strength at short distances $r$ from the electrode is multiplied with the factor

$$
f(r)=c \cdot \frac{a^{3}}{r^{3}}+1
$$

matching the field at the tip of ellipsoids (are at the tip of half of an ellipsoid sitting on a infinite surface) quite well, where $a$ is the smaller semi-axis of the ellipsoid and $c$ given by the relative of both semi-axis. For the following calculations (and in agreement with the surface roughness measurement), $a$ is set to $5 \mu \mathrm{m}$ and $c=8$ (representing a second semi-axis $b \sim 2.5$ ). Further it is assumed that their frequency of occurrence is about $0.25 \%$. In the case of negative polarity, field emission is not triggered at this field strengths, whereby a further field enhancement by a factor 3 by microprotrusions at the surface of the ellipsoids is considered in the calculation.

\section{RESULTS}

In the following sections, the results of the breakdown experiments are presented. Each breakdown probability curve is the result of $150-600$ shots. Indicated polarity in the description of the results always refers to the contact side with higher field stress (higher field inhomogeneity).

\subsection{FIXED CONTACT SYSTEM WITH LI STRESS}

In order to investigate the breakdown behavior of an individual contact system, breakdown experiments were carried out using the fixed contact system and a plane electrode. The goal was to investigate whether the breakdown behavior (or probability) of both contacts could be considered individually or not. For this, the breakdown probability was measured separately for the single arcing and main contact and then compared to the measured breakdown probability for the combined contact system with either the same distance between contacts and the plane electrode or approximately the same electric field stress as in the single contact experiments. This can be seen in Figure 6 showing schematic drawings of these electrode arrangements together with the electric field along the shortest distance between the electrodes. The setups (a)-(c) in Figure 6 have the same contact distance of $30 \mathrm{~mm}$ whereas in setup (d), the arcing contact tip is exposed by $6 \mathrm{~mm}$ with respect to the nominal contact. Notably, the electric field between the main contact and the plane electrode remain unchanged for setups (b)-(d), whereas the field between arcing contact and plane electrode is significantly different comparing setup (a) with (c). In the combined contact setup (d) (equal field arrangement) the distance was chosen so that the maximum electric fields at both contacts were comparable with the single contact setups (a) and (b).
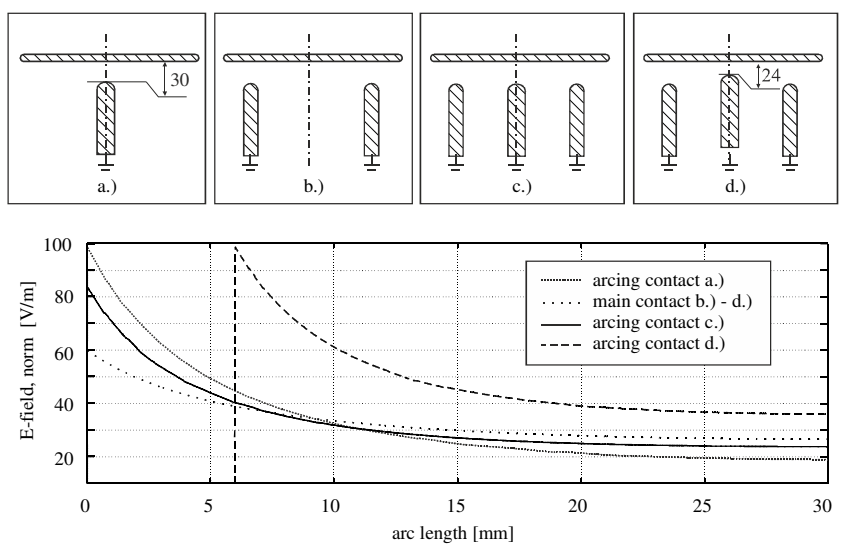

Figure 6. Electrode arrangements and corresponding electric field along shortest distance between the electrodes, calculated with $1 \mathrm{~V}$ potential difference: a.) arcing contact and b.) main contact vs. plane electrode. c.) and d.) are combined contact systems vs. plane electrode with equal contact distance as in case of the single contacts c.) and with equal maximum electric field d.).

The breakdown probability curves with standard LI voltages are shown for both polarities in Figure 7. Further, the measured $50 \%$ breakdown voltages $U_{50}$ are summarized in Table 1 together with the calculated breakdown voltages $U_{\text {calc }}$ using the described streamer criterion for negative polarity and the volume-time criterion for positive polarity.

The arrangement a.), arcing contact vs. plane electrode showed the lowest breakdown voltage with $U_{50}=485 \mathrm{kV}$ for negative polarity and $U_{50}=598 \mathrm{kV}$ for positive polarity. The theoretical $50 \%$ breakdown voltage calculated with the streamer criterion and field emission is $U_{50}=498 \mathrm{kV}$. The calculation of the volume-time criterion results in $U_{50}=654 \mathrm{kV}$.

The highest breakdown voltages were measured with the arrangement b.), main contact vs. plane electrode having a $50 \%$ breakdown voltage of $U_{50}=724 \mathrm{kV}$ (negative) and $U_{50}=792 \mathrm{kV}$ (positive), respectively. For this configuration, 
the theoretical breakdown voltage is calculated to be $\mathrm{U}_{50}=731 \mathrm{kV}$ (field emission, i.e. negative) and $817 \mathrm{kV}$ (volume-time, i.e. positive).

In the equal distance arrangement (c), the resulting electric field between the main contact and plane remains unchanged, whereas the arcing contact is partly shielded by the main contact and the maximum electric field on the contact tip is reduced by $\sim 15 \%$. Due to the decreased maximum electric field on the arcing contact in arrangement (c) compared to (a), a higher breakdown voltage resulted with $U_{50}=587 \mathrm{kV}$ (negative) and $U_{50}=774 \mathrm{kV}$ (positive). The theoretical breakdown voltage is $U_{50}=596 \mathrm{kV}$ for the plug (field emission) and $734 \mathrm{kV}$ (volume-time) and $809 \mathrm{kV}$ and $820 \mathrm{kV}$ for the plug and the ring respectively. For negative polarity, the breakdown probability curves of the individual contact (b) does not overlap with that of the combined contact system (c) and all breakdowns occurred on the plug. This is different for positive polarity. Here, breakdown probability curves (b) and (c) overlap and of the 234 breakdowns (in a series of 312 shots), 54 breakdowns occurred also on the main contact. Figure 8 (left) shows the location of the arc roots reconstructed from the photographs of the breakdown. It can be clearly seen that the arc roots are homogeneously distributed across the surface.
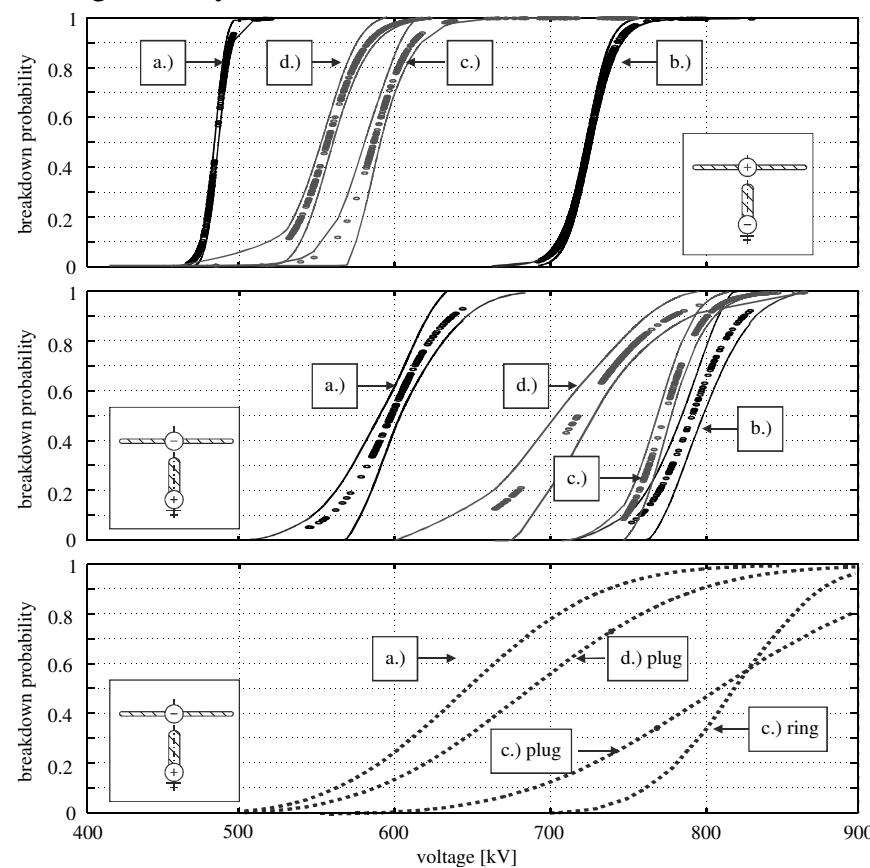

Figure 7. Breakdown probability curves for the four electrode arrangements a.) - d.) according to Figure 6. The electrode system was stressed with lightning impulse voltage of negative polarity (top) and positive polarity (middle) on the inhomogeneous contact side. The bottom picture shows the calculated breakdown probability curves (positive polarity) for indicated contacts.

As mentioned above, the equal field arrangement (d) was chosen to have comparable maximum electric field stresses at the main and arcing contact compared to their single arrangements. Nonetheless, the measured breakdown probability curves from (d) did not overlap with those of (a) (the lower breakdown voltage of the two single contacts).
The resulting breakdown voltages were measured to be $U_{50}=557 \mathrm{kV}$ (negative) and $U_{50}=725 \mathrm{kV}$ (positive), while the theoretical breakdown voltage is $U_{50}=507 \mathrm{kV}$ and $686 \mathrm{kV}$ and is thus comparable to configuration (a). At negative polarity, all breakdowns occurred on the plug. For positive polarity, out of the 146 breakdowns (in a series of 208 shots), one occurred on the main contact having a peak amplitude of $758 \mathrm{kV}$ and a time to breakdown of $4.46 \mu \mathrm{s}$.
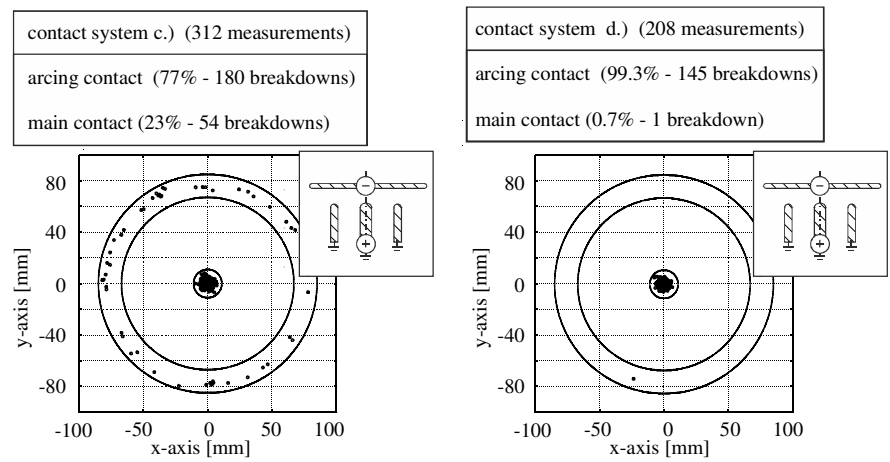

Figure 8. Arc root point distribution on main and arcing contact when tested with positive lightning impulse voltage. In the equal distance arrangement (c), almost a quarter of all breakdowns occurred on the main contact. In the equal field arrangement (d), main contact breakdown probability was significantly reduced but not eliminated.

Table 1. Measured $\left(U_{50}\right)$ and calculated $\left(U_{\text {calc }}\right)$ breakdown voltages of arrangement (a) - (d) (see Figure 6) for positive (+) and negative (-) polarity. Breakdown voltages are calculated using the ideal streamer criterion (negative) or the volume-time criterion (positive). Additionally the ratio between calculated and measured values is given.

\begin{tabular}{|c|c|c|c|c|c|c|}
\hline & \multicolumn{3}{|c|}{$(-)$} & \multicolumn{3}{|c|}{$(+)$} \\
\hline$[\mathrm{kV}]$ & $U_{50}$ & $U_{\text {calc }}$ & ratio & $U_{50}$ & $U_{\text {calc }}$ & ratio \\
\hline (a) & 485 & 498 & 0.97 & 598 & 645 & 0.93 \\
\hline (b) & 724 & 731 & 0.99 & 792 & 817 & 0.97 \\
\hline (c) arc & \multirow{2}{*}{587} & 596 & 0.98 & \multirow{2}{*}{774} & 809 & 0.96 \\
\hline main & & 734 & no BD & & 820 & 0.94 \\
\hline (d) arc & \multirow{2}{*}{557} & 507 & 1.09 & \multirow{2}{*}{725} & 686 & 1.06 \\
\hline main & & 735 & no BD & & 821 & one $\mathrm{BD}$ \\
\hline
\end{tabular}

Figure 9 shows the measured time to breakdown values for arrangement (a) - (d) and both polarities. It can be seen that the time to breakdown is always higher than the time to peak of the impulse voltage form, indicating that all breakdowns happened after the peak voltage. The breakdown voltage is generally lower for negative polarity, whereas its spread of time to breakdown is larger. Further, negative polarity generally shows a stronger increase of breakdown voltage towards smaller times.

\subsection{FULL CONTACT SYSTEM COMPARING LI WITH VFT VOLTAGE STRESS}

In order to investigate the influence of the voltage shape on the breakdown location, the contact system according to the schematic drawing in Figure 10 was tested. The relative distance of the four contacts was chosen to provoke an electric field distribution comparable to real high voltage gas circuit breakers. On the moving contact side, the arcing contact (tulip) is exposed in axial direction by $3 \mathrm{~mm}$ 
compared to the main contact. On the fixed contact side, in turn, the plug is withdrawn by $5 \mathrm{~mm}$. Despite this, the maximum electric field strength on the plug is $\sim 75 \%$ higher than on the tulip. The fixed contact side is thus the more inhomogeneous one and decisive for breakdown initiation. The theoretical breakdown voltages calculated by the streamer criterion are $U_{50}=393 \mathrm{kV}$ for the arcing contact and $U_{50}=647 \mathrm{kV}$ for the main contact. The ratio of the maximum electric field between arcing and main contact (coordination factor $C$ ) was $\sim 1.8$ for the fixed contact side and $\sim 1$ for the moving contact side. Comparing the maximum electric fields along the shortest distance between the electrodes (as in Figure 10, right), the ratio was $\sim 1.66$ and $\sim 0.87$ respectively.

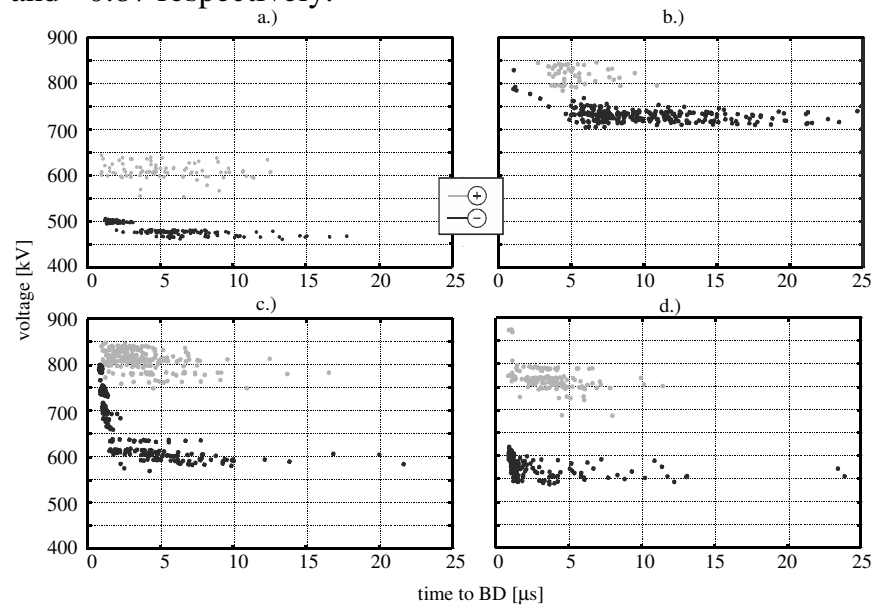

Figure 9. Time to breakdown measurements for electrode arrangements (a) - (d) (see Figure 6).

This geometry was tested with standard LI voltage stress (disconnector closed) and with superimposed VFT (disconnector opened). Although the measurement setup was not calibrated for high frequency measurements, an FFTAnalysis of the measured signals as shown in Figures 4 and 5 was performed. This analysis revealed that the LI impulse voltage is confined to a frequency up to approximately $500 \mathrm{kHz}$ whereas most of the energy of the VFT signals is within $0-58 \mathrm{MHz}$ with further components up to $156 \mathrm{MHz}$. Therefore there is a clear distinction of the two voltage waveforms concerning their frequency content.
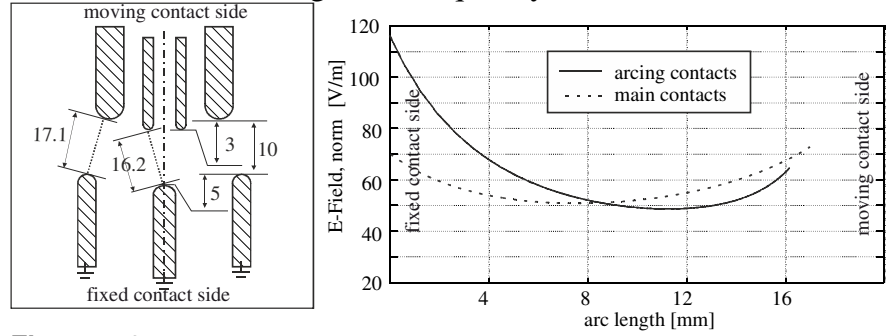

Figure 10. Complete contact system representing high voltage circuit breaker contacts. Different relative contact separations were chosen, resulting in a low coordination on the moving contact side and a high coordination on the fixed contact side. The electric field calculation was performed with $1 \mathrm{~V}$ potential difference between the electrodes.
The corresponding measured breakdown probability curves are shown in Figure 11 for LI and VFT voltage of both polarities. In line with the previous results, negative polarity showed lower breakdown voltages having $U_{50}=426 \mathrm{kV}$ for lightning impulse and $U_{50}=340 \mathrm{kV}$ (prospective) and $394 \mathrm{kV}$ (maximum) if VFT was superimposed. At positive polarity the $50 \%$ breakdown voltages were $U_{50}=588 \mathrm{kV}, \quad 530 \mathrm{kV}$ and $574 \mathrm{kV}$, respectively. The calculated breakdown voltage using the streamer integral respecting field emission is $393 \mathrm{kV}$ and $536 \mathrm{kV}$ using the volume-time criterion, assuming the plug is the decisive electrode.

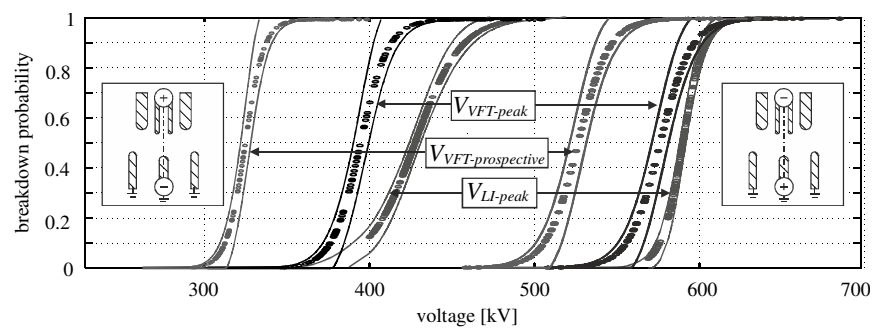

Figure 11. Breakdown probability curves for complete contact system and both polarities, when stressed with lightning impulse voltage shape $\left(\mathrm{V}_{\text {LI-peak }}\right)$ and with VFT voltage superimposed on lightning impulse ( $\mathrm{V}_{\mathrm{VFT} \text {-peak }}$ ) see Figure 4 and Figure 5. The amplitude of the prospective LI impulse voltage shape, which excited the VFT voltage, is referred to as $\mathrm{V}_{\mathrm{VFT} \text {-prospective. }}$

When tested with LI negative polarity, all breakdowns occurred on the arcing contact, whereas at positive polarity a distribution of breakdowns on both contacts was detected (cf. Figure 12). Main contact breakdown happened 9 times out of 255 breakdowns (in a series of 375 shots) when tested with lightning impulse voltage shape. At the superposition of VFT, an increase in main contact breakdown was recorded with 63 out of 91 breakdowns (in a series of 160 shots). In Figure 12, a concentration of arc root points on one quarter of the ring electrode is visible. A slight misalignment between the fixed contact and the plane electrode could be identified as the cause. Nonetheless, the comparison between LI and VFT experiments was still valid because no change of the experimental setup was made for the entire measurements series, except for the change of applied voltage shape.
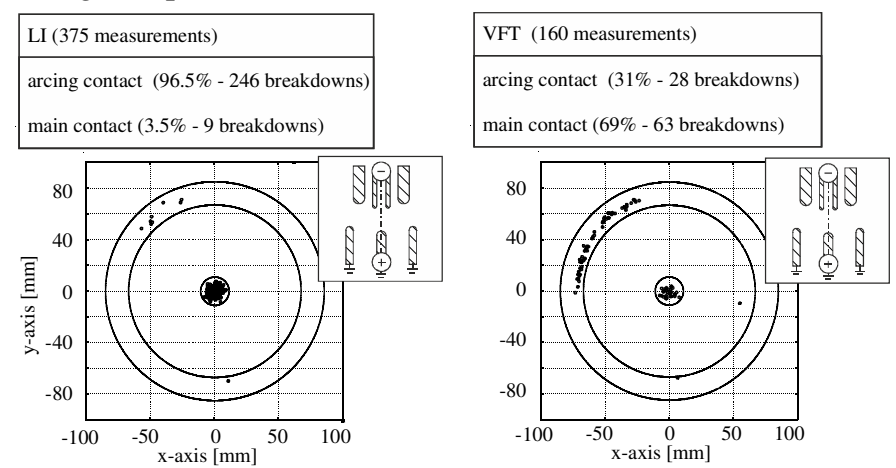

Figure 12. Arc root point distribution when testing with positive polarity on inhomogeneous contact side. The experiments with lightning impulse show a much lower main contact breakdown probability (3.5\%) compared to the same arrangement tested with VFT-voltage (69\%). 
The measured and calculated breakdown voltages are summarized in Table 2. Since each of the four contacts composing the contact system may have an influence on the breakdown behavior, the breakdown voltage was calculated for each contact individually respecting the voltage polarity, i.e. streamer (neg) and volume-time (pos). For the latter the corresponding voltage shape (LI or VFT) was used.

Table 2. Measured and calculated LI and VFT (see Figure 4 and Figure 5) breakdown voltages for negative a) and positive b) polarity. $U_{\text {calc }}$ denotes the calculated breakdown voltage using the ideal streamer criterion (s) or the volume-time criterion (v) dependent on whether the resulting stress on the single electrode is of negative or positive polarity.

a)

\begin{tabular}{|c|c|c|c|c|c|c|}
\hline \multicolumn{2}{|c|}{ Negative } & \multicolumn{2}{|c|}{ Lightning Impulse } & \multicolumn{3}{|c|}{$V F T$} \\
\hline & {$[\mathrm{kV}]$} & $U_{50}$ & $U_{\text {calc }}$ & $\begin{array}{c}U_{50} \\
\text { (prosp) }\end{array}$ & $\begin{array}{c}U_{50} \\
(\max )\end{array}$ & $U_{\text {calc }}$ \\
\hline \multirow[b]{2}{*}{ ప } & fixed & \multirow{4}{*}{426} & $393(\mathrm{~s})$ & \multirow{4}{*}{340} & \multirow{4}{*}{394} & $444(\mathrm{~s})$ \\
\hline & moving & & $802(\mathrm{v})$ & & & $935(\mathrm{v})$ \\
\hline \multirow{2}{*}{ 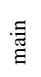 } & fixed & & $647(\mathrm{~s})$ & & & $731(\mathrm{~s})$ \\
\hline & moving & & $692(\mathrm{v})$ & & & $806(v)$ \\
\hline
\end{tabular}

b)

\begin{tabular}{|c|c|c|c|c|c|c|}
\hline \multicolumn{2}{|c|}{ Positive } & \multicolumn{2}{|c|}{ Lightning Impulse } & \multicolumn{3}{|c|}{$V F T$} \\
\hline & {$[\mathrm{kV}]$} & $U_{50}$ & $U_{\text {calc }}$ & $\begin{array}{c}U_{50} \\
\text { (prosp) }\end{array}$ & $\begin{array}{c}U_{50} \\
(\max )\end{array}$ & $U_{\text {calc }}$ \\
\hline \multirow{2}{*}{ है } & fixed & \multirow{4}{*}{588} & $536(\mathrm{v})$ & \multirow{4}{*}{530} & \multirow{4}{*}{574} & $626(\mathrm{v})$ \\
\hline & moving & & $648(\mathrm{~s})$ & & & $732(\mathrm{~s})$ \\
\hline \multirow{2}{*}{ 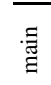 } & fixed & & $734(v)$ & & & $853(\mathrm{v})$ \\
\hline & moving & & $614(\mathrm{~s})$ & & & $693(\mathrm{~s})$ \\
\hline
\end{tabular}

\section{DISCUSSION}

The measurements presented in this paper indicated that the major breakdown criterion for low inhomogeneous electrode arrangements under impulse voltage stress was the occurrence of a first electron. This condition was fulfilled more easily if the high field electrode was on negative polarity. The necessary electric field values to trigger electron field emission are likely to be reached in the present application, taking the macroscopic electric fields (cf. Figures 6 and 10) at the measured breakdown voltages and the field enhancement due to surface roughness into account. This results in an overall lower breakdown voltage at negative polarity. The time to breakdown curves (Figure 9) show a minimum required time to breakdown of approximately $1 \mu \mathrm{s}$, but a generally wider spread compared to positive polarity. It is assumed that an electron is present as soon as the necessary field value for electron emission is reached. But the time scales of the negative formative time lag are larger than those at positive polarity [13]. The lower electric fields in the case of negative polarity (due to lower breakdown voltages), may further increasing the negative formative time lag [10]. At positive polarity, the formative time-lag is smaller and the time to breakdown is dominated by the statistical time-lag.

Consequently, the breakdown voltages, i.e. breakdown probability curves, can be calculated by applying the streamer criterion for negative polarity and the volume-time criterion for positive polarity. In the present application this could explain the difference between measurement and prediction.

Although the calculated breakdown voltages are not precise, an overlap of the breakdown probability curves in arrangement (c) and (d) (cf. Figure 7) could be reproduced through the application of the volume-time criterion (small difference of the voltage values in Table 1). Whereas for negative polarity a coordination factor of $C=1.4$ and 1.63 for arrangement (c) and (d) was sufficient to prevent main contact breakdown, it failed at positive polarity. Especially noticeable are the measured breakdown voltages, which are larger for both polarities on the equal field arrangement (d) than on the arcing contact arrangement a.). Clearly, only the maximal field strengths are the same between (a) and (d), but because of the shorter contact separation one would expect lower breakdown voltages in arrangement (d) at first sight. One reason for the contrary behavior is certainly the fact that, apart from the maximal field strengths, the fields are generally smaller in the equal field arrangement (d) than in arrangement (a) (e.g. due to the shorter distance from the plug to the plane, the distribution of surface gradients is less homogeneous). Therefore, the surface for field emission (negative polarity) and the critical volume $V_{\mathrm{cr}}$ (positive polarity) are smaller in the equal field arrangement d.) compared to arrangement a.). Indeed, this circumstance is reflected in the higher calculated positive breakdown voltage from arrangement (d) of $686 \mathrm{kV}$ compared to $645 \mathrm{kV}$ of arrangement (a) (see Table 1). However, this difference does not seem to explain the whole increase of breakdown voltage from arrangement a.) to the equal field arrangement (d), for which no complete explanation can yet be given.

Therefore, the initial question - whether the experimental evaluation of single contact breakdown (with approximately the same fields) is sufficient to predict the breakdown behavior of the full contact system and to ensure that only arcing contact breakdown occurs - can only be answered in the negative.

Similarly, in the full contact arrangement (Figure 10) a coordination factor of $C=1.81$ is sufficient to prevent main contact breakdown at negative polarity, but not at positive polarity. Considering the calculated breakdown voltages (cf. Table 2) of all four contacts, the measured main contact breakdown can be explained as follows: The electric field is maximal at the fixed arcing contact, which is stressed with positive polarity, calculated to be $536 \mathrm{kV}$. The electric field at the moving (main and arcing) contacts is significantly lower, but they are stressed with negative polarity, which has a lower breakdown voltage of $614 \mathrm{kV}$ and $648 \mathrm{kV}$, respectively. Considering the widths of the breakdown probability curves, the initiation of the breakdown may thus also be on the moving contact side. This may explain the measured overall breakdown probability of $3.5 \%$ on the main contacts, although the maximum electric field at the arcing contact is $\sim 70 \%$ percent higher.

When the same contact system was stressed with VFT voltage, a dramatic decrease in breakdown voltage and an increase in main contact breakdowns could be observed. It 
seemed likely that the increased voltage stress at the contacts due to constructive reflection of the incident travelling wave and the additional heating of the streamer region by the highfrequency mechanism was responsible for the decrease in (prospective) breakdown voltage (cf. Figure 11).

The frequency content of the voltage signal seems sufficient to trigger the high frequency breakdown mechanism [15]. However, a rough estimation of the stray capacitances of arcing and main contact predicted only small differences between them, making the high-frequency mechanism an unlikely explanation for the increase in main contact breakdown probability.

If the volume-time criterion is applied on VFT-voltages, a strong rise in breakdown voltages of the whole contact system results from the calculations (Table 2). Due to the fast oscillating voltage form (with lower voltages in the average than with pure LI), the occurrence of a first electron initiating a discharge becomes less likely. Again, this fact applies only to the fixed contact side (being on positive polarity). Similarly to the explanation of main contact breakdown for the positive LI experiments above, the breakdown values have to be compared with the moving main contact on negative polarity, whereas the difference between the two breakdown values decreased $(626 \mathrm{kV}$ and $693 \mathrm{kV})$.

In the present investigations, after each voltage application, an ac voltage was applied on the contact systems, followed by a fixed time period to the next voltage application, to ensure a comparable ion density distribution between the contacts in all experiments. This is not equivalent to the IP density in equilibrium, reached after break times of several minutes $[11,13]$. Obviously, the IP density influences (in the case of volume-time-criterion, i.e. positive polarity) the breakdown probability decisively; the higher the ion densities, the lower are the breakdown voltages. In nominal service of circuit breakers low IP densities are to be expected.

\section{CONCLUSION}

Even though the investigated specific contact arrangements does not permit conclusion to generality, the understanding of breakdown behavior of low inhomogeneous electrode arrangements in $\mathrm{SF}_{6}$ could be extended through the results presented in this work:

- Clearly, dielectric coordination based on defining a maximal surface gradient is not sufficient if dimensioning high voltage circuit breakers toward minimal size and coordination factor.

- The breakdown voltages calculations based on streamer integral and volume-time criteria yield convincing results, especially in connection with coordinating multi-electrode arrangements. However, the calculated values are too large when compared with the experiment. Further, the measured difference in breakdown voltage from single contact to multi-contact measurements with approximately the same field strengths can only be partially reproduced by the calculation methods.
- Therefore, it must be concluded that high voltage circuit breaker contact systems should be tested as a whole and not through extrapolation from single contact experiments.

- Particular attention has to be paid to the dependence of breakdown voltages on the polarity. With regard to high voltage circuit breaker design, it is recommended to implement proper coordination factors on both sides of the breaker contacts (moving and fixed side). Coordination factors being implemented only on one side, make the breaker prone to positive over voltages applied on the coordinated side, as the contacts on the other side of the breaker, being on negative polarity, tend to lower breakdown voltages.

- Further it is not possible to conclude from breakdown probabilities for a certain voltage waveform to the breakdown probabilities of another voltage waveform. Therefore, the occurrence of VFT voltages, potentially the case in circuit breakers with more than one interrupter unit in series where pre- or re-ignition excites travelling waves in the breaker, may further accentuate coordination difficulties.

- Clearly, the results cannot be directly generalized to contact systems of real breakers, with problems such as contact finger edges (with less surface for field emission or the volume-time-criterion), roughened surfaces after arcing or by a macroscopic change (i.e. shortening by arcing reducing the field stress). However, it has been shown that there are effects which require an analysis beyond the macroscopic field simulation or the measurement of individual contacts only, when coordinating new designs. The developer has to take this into account by using the recipe shown in this paper.

\section{ACKNOWLEDGMENT}

Financial support of the project from ABB Switzerland, Ltd. (Corporate Research and High Voltage Products) is gratefully acknowledged. Some of the measurements were supported by students in the framework of their semester or master thesis.

\section{REFERENCES}

[1] F. Pinnekamp, "The circuit breaker", ABB Review, Vol. 1, pp. 75-78, 2007.

[2] C. Flurscheim, Power Circuit Breaker Theory and Design, $2^{\text {nd }}$ ed. Peter Peregrinus Ltd., Chapter 1, 1982.

[3] P. Simka, Dielectric Coordination of High Voltage Gas Circuit Breakers, Ph.D. Thesis, ETH Zurich Switzerland, 2011.

[4] M. Beyer, W. Boeck, K. Möller and W. Zaengl, Hochspannungstechnik, Springer Verlag, 1986, Chapter 4.

[5] W. Mosch and W. Hauschild, Hochspannungsisolierungen mit Schwefelhexafluorid, Dr. Alfred Hüthig Verlag, Heidelberg, ISBN 37785-0540-8, Chapters 2 and 4, 1979.

[6] W. Boeck and J. Kindersberger, "Determination of the statistical time lag in $\mathrm{SF}_{6}$ “, $4^{\text {th }}$ Int'l. Sympos. High Voltage Eng. (ISH), Athens, Greece, Paper 31.06, pp. 1-4, 1983.

[7] N. Wiegart, L. Niemeyer, F. Pinnekamp, W. Boeck, J. Kinderberger, R. Morrow, W. Zaengl, M. Zwicky, I. Gallimberti and S. Boggs, "Inhomogeneous Field Breakdown in GIS - Part II", IEEE Trans. Power Del., Vol. 3, pp. 931-938, 1988

[8] W. Boeck, "SF6 insulation breakdown behavior under impulse stress", in K. Ragaller (ed.), Surges in High-Voltage networks, Plenum Press, New York, USA, 1979. 
[9] E. Kuffel, W.S. Zaengl and J.Kuffel, High Voltage Engineering Fundamentals, 2nd Ed. Elsevier, ISBN 978-0-7506-3634-6, Chapter 5, 2000.

[10] M. Seeger, L. Niemeyer and M. Bujotzek, "Partial discharges and breakdown at protrusions in uniform background fields in SF6", J. Phys. D: Appl. Phys., Vol. 41, 185204, 2008.

[11] J. Kindersberger, The Statistical Time-lag to Discharge Inception in $S F_{6}$, Ph.D. Thesis, TU Munich, Germany, 1980.

[12] W. Boeck, "Volumen-Zeit-Gesetz beim Stossspannungsdurchschlag von SF6", ETZ-A Vol. 96, No. 7, 300-305, 1975.

[13] L. Niemeyer, L. Ullrich and N. Wiegart, "The mechanism of leader breakdown in electronegative gases", IEEE Trans. Electr. Insul., Vol. 24, pp. 309-324, 1989.

[14] H. Hiesinger, "Leader breakdown for inhomogeneous fields in case of VFT conditions“", $7^{\text {th }}$ Int'l. Sympos. High Voltage Eng. (ISH), Dresden, Germany, pp.67-70, 1991.

[15] H. Hiesinger, Der Hochfrequenz-Durchschlagsmechanismus in $\mathrm{SF}_{6}$ bei Schwingenden Transienten Überspannungen, Ph.D. Thesis, TU Munich, Germany, 1991.

[16] "IEC 60060-1, High-voltage test techniques - Part 1: General definitions and test requirements", International Electrical Committee IEC, Geneva, 2006.

[17] W. Hauschild and W. Mosch, Statistical Techniques for High-voltage Engineering, Peter Peregrinus Ltd., Vol. 1, Chapters 2 and 5, 1992.

[18] P. Simka nd C. M. Franck, "Measuring breakdown probabilities of $\mathrm{SF}_{6}$ insulated, low inhomogeneous electrodes", XVIII ${ }^{\text {th }}$ Int'l. Conf. Gas Discharges and Their Application, Greifswald, Germany, pp. 610-613, 2010.

[19] X. Xu, S. Jayaram and S.A. Boggs, "Prediction of Breakdown in SF6 under Impulse Conditions", IEEE Trans. Dielectr. Electr. Insul., Vol. 3, pp. 836-842, 1996.

[20] P. Simka, "An Approach to Model Very Fast Transients in High Voltage Circuit Breakers“, IEEE Int'l. Sympos. Electr. Insul. (ISEI), pp. 449-452, 2008.

[21] P. Simka, "A Complete Circuit Breaker Model for Calculating Very Fast Transient Voltages“, IEEE Int'l. Sympos. Elecr. Insul. (ISEI), pp. $1-5,2010$

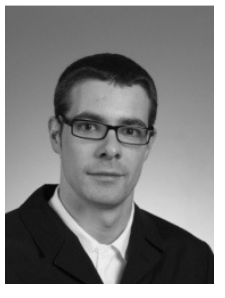

Philipp Simka was born in Chur, Switzerland in 1978. He received the M.Sc. degree from the Department of Electrical Engineering and Information Technology, ETH Zurich, Switzerland in 2005 and is currently doing his Ph.D. degree at the Institute for Power Systems and High Voltage Technology, ETH Zurich, Switzerland. His research interests cover dielectric phenomena, high voltage test techniques and high frequency phenomena associated with high voltage equipment.

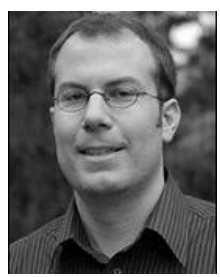

Ueli Straumann (M'10) was born in Aarau, Switzerland in 1975. He received a diploma in theoretical physics from the University of Zurich, Switzerland in 2001 and the Ph.D. degree from ETH Zurich, Switzerland, in 2007. Since then he has been Senior Assistant and Lecturer in the High Voltage Laboratory of ETH Zurich. His research interests include corona of OHL, the description of ion flow fields and gaseous insulation in general.

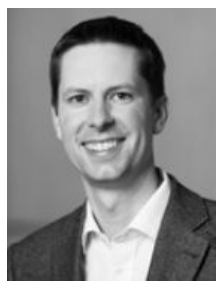

Christian M. Franck (M'04-SM'11) received a diploma in physics from the University of Kiel, Germany in 1999 and the Ph.D. degree in physics from the University of Greifswald, Germany in 2003. He was with the Swiss corporate research center of ABB from 2003-2009 as a Scientist and Group Leader for gas circuit breakers and high-voltage systems. Currently, he is an /Assistant Professor for High Voltage Technology at the Swiss Federal Institute of Technology (ETH), Zurich, Switzerland. 